\title{
RailSLAM
}

\section{Localization of Rail Vehicles and Mapping of Geometric Railway Tracks}

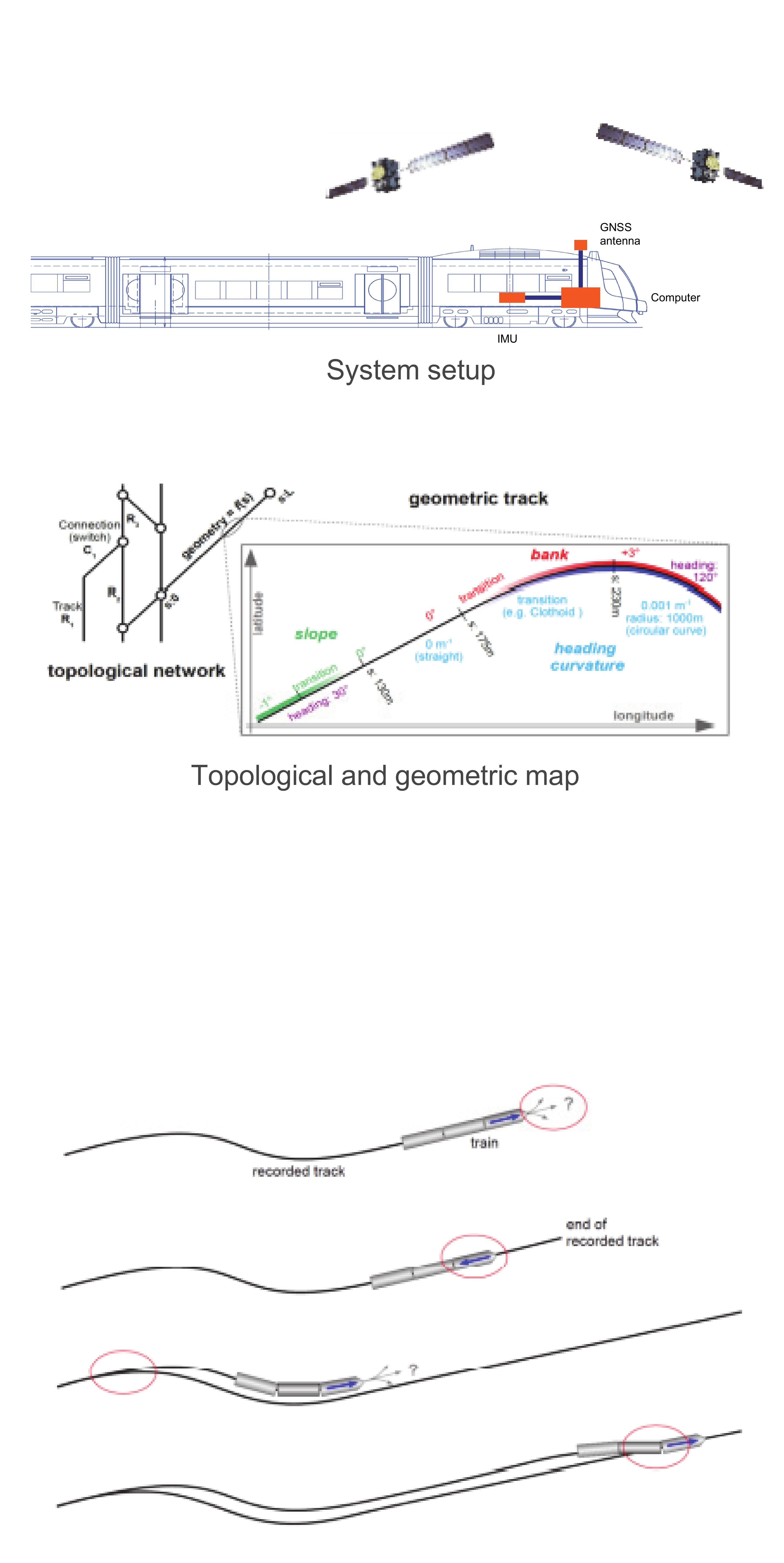

SLAM scenarios

\section{Train Localization}

7 Safety critical applications (Collision Avoidance)

7 onboard sensors: GNSS + IMU

7 Topological-geometric map enables onboard train localization

7 Measurement of train kinematics (IMU), model contains track geometry (map)

$$
\begin{aligned}
& \omega^{x}=C_{\phi} \dot{s} \quad-C_{\psi} \dot{s} \dot{s i n} \theta \\
& \omega^{y}=C_{\theta} \dot{s} \quad \cdot \cos \phi+C_{\psi} \dot{s} \dot{s i n} \phi \cos \theta \\
& \omega^{2}=\underbrace{C_{\psi} \dot{s}} \underbrace{\cdot \cos \theta \cos \phi-C_{\theta} \dot{s} \sin \phi} \\
& \underbrace{c_{\substack{\text { attitiude } \\
\text { corrections }}}}_{\substack{\text { major } \\
\text { uur raters }}} \\
& a^{x}=g \sin \theta+\overbrace{s}^{\text {traction }} \\
& a^{y}=-g \sin \phi \cos \theta+C_{\psi} \dot{s}^{2} \cos \phi-C_{\theta} \dot{s}^{2} \sin \phi \\
& a^{2}=\underbrace{-g \cos \phi \cos \theta}_{\text {gravity }}-\underbrace{C_{C_{b} \dot{s}^{2}} \sin \phi}_{\text {croses }}-\underbrace{C_{\theta} \dot{s}^{2} \cos \phi}_{\text {vertical }}
\end{aligned}
$$

SLAM for Railways

1. White space mapping

2. SLAM on prior map

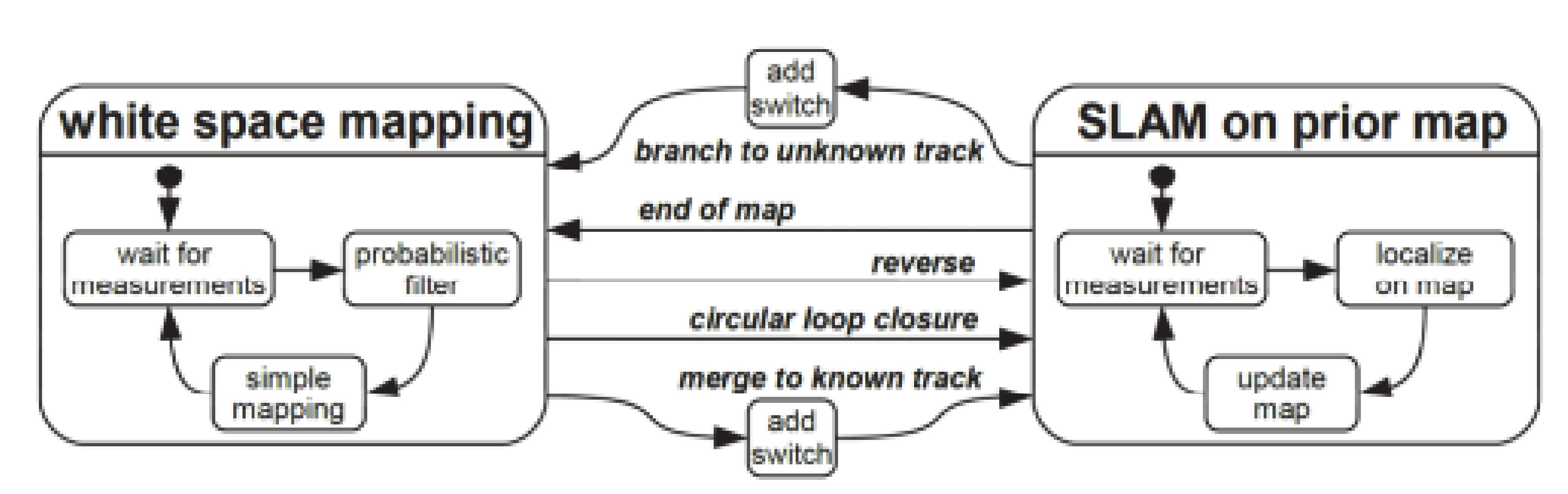

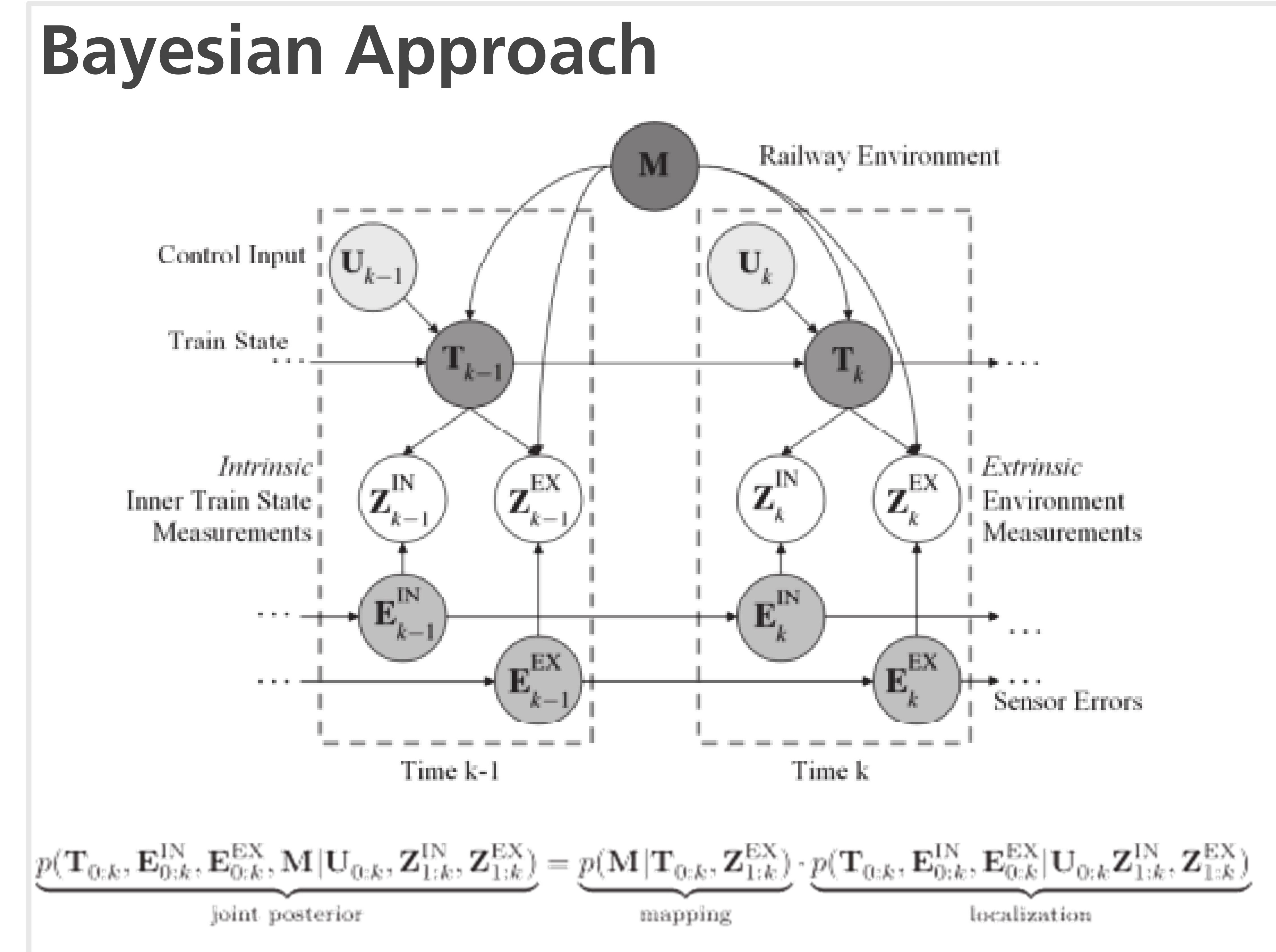

Rail Vehicle Filter

$>$ Train state estimation by EKF

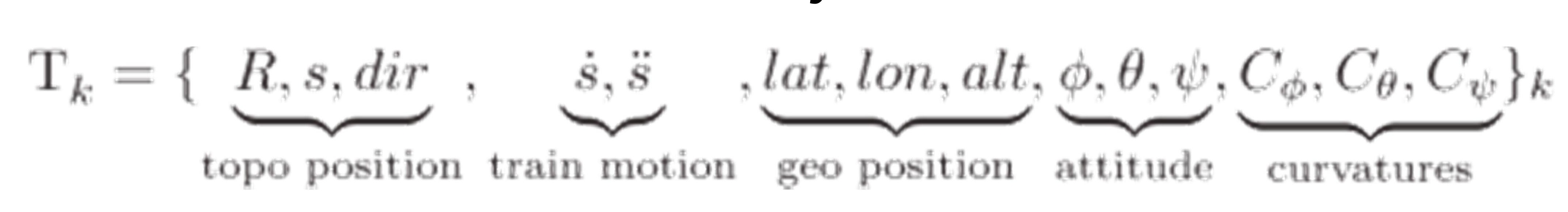

\section{Train Measurements}

7 Industrial tracks of inland port

7 GNSS (Septendrio-GPS) and MEMS-IMU (Xsens)

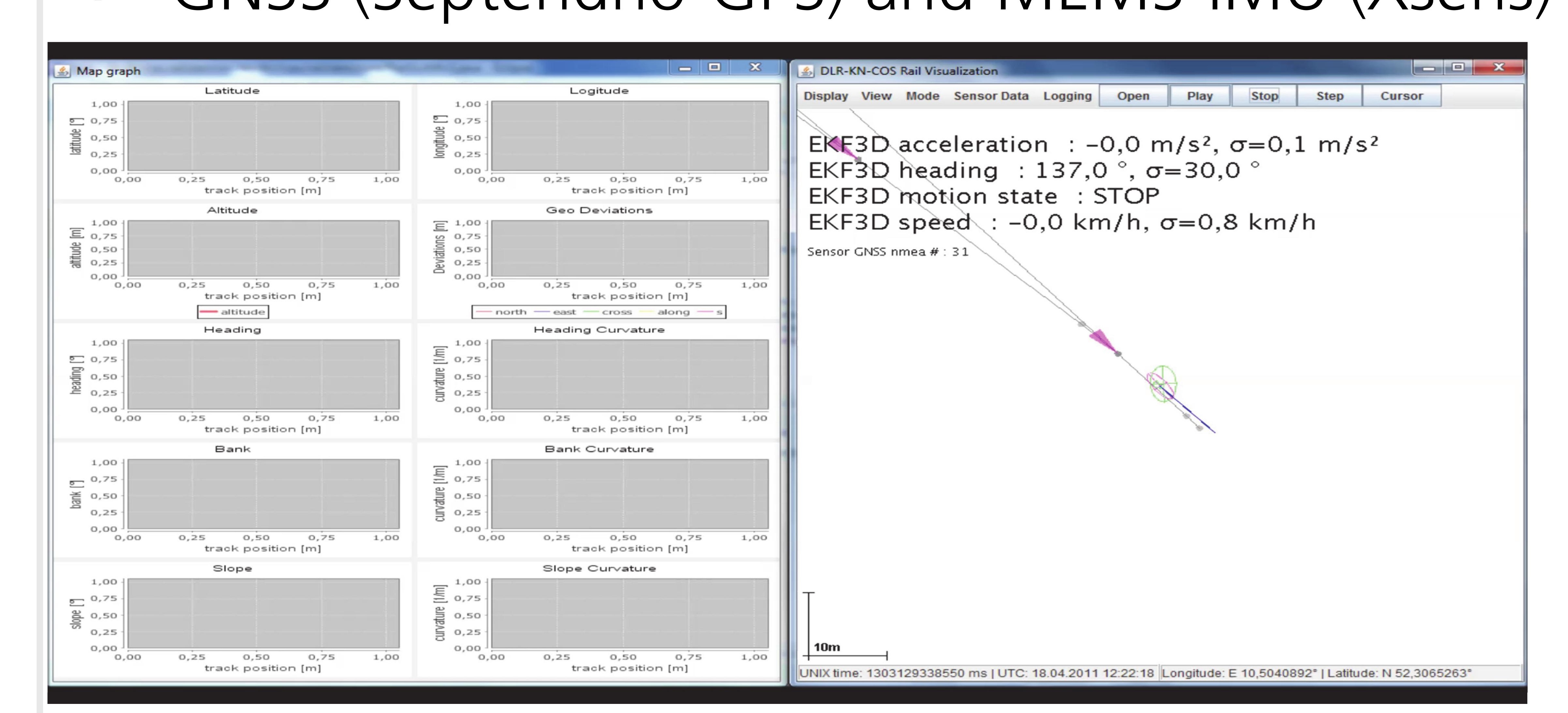

Probabilistic geometric feature-rich map

7 Track point contains geometry and variances parameterized on track length (1D position)

$$
\mathrm{Tp}_{s}=\{\underbrace{s}_{\text {1D position }}, \underbrace{\text { lats }_{s} \text { lon }_{s}, \text { alt }_{s}}_{\text {geo position }}, \underbrace{\phi_{s}, \theta_{s}, \psi_{s}}_{\text {attitude }}, \underbrace{\left.C_{\phi, s}, C_{\theta, s}, C_{\psi, s}\right\}}_{\text {curvatures }}
$$

$>$ Estimated map of RailSLAM:

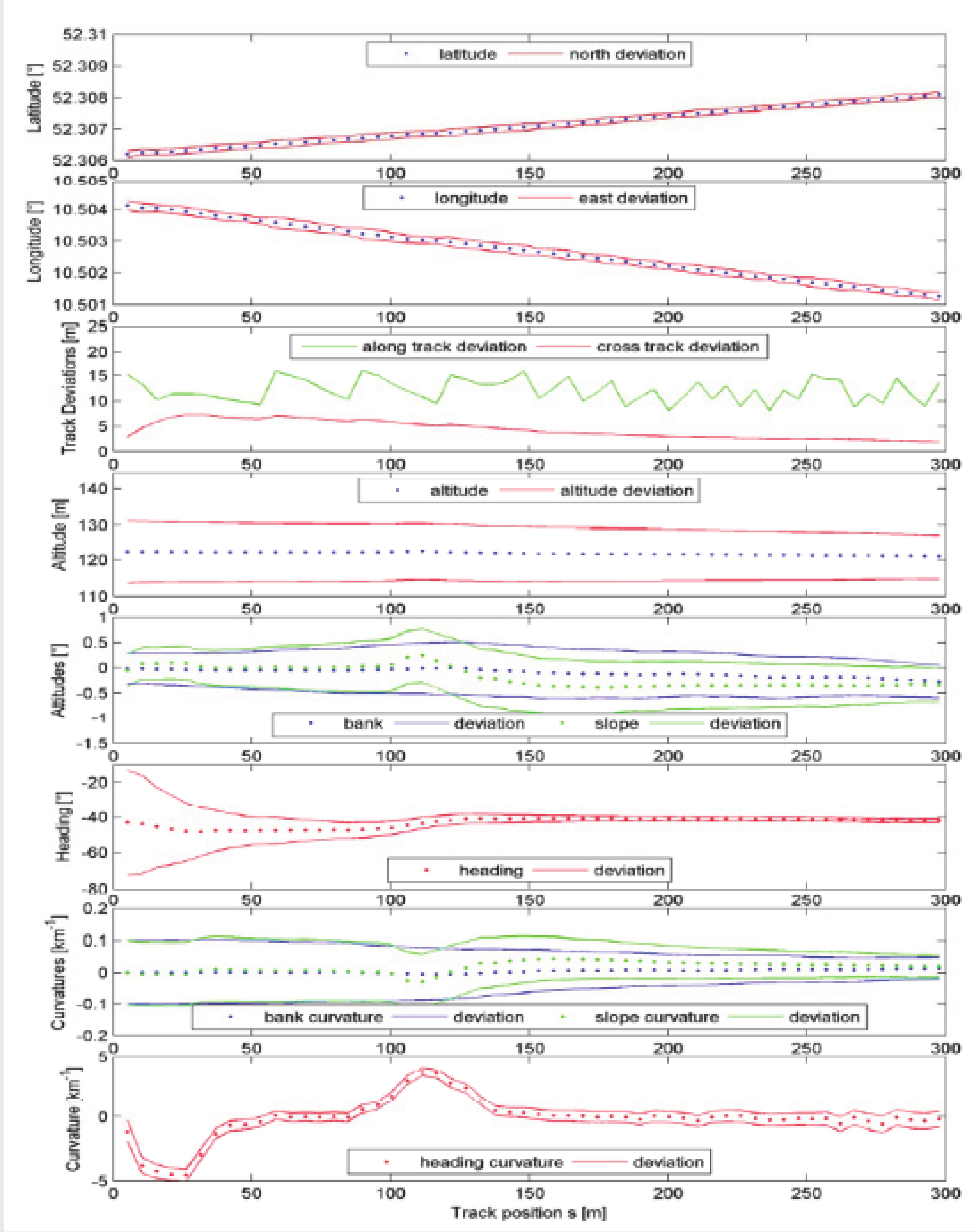




\section{RailSLAM - \\ Localization of Rail Vehicles and Mapping of Geometric Railway Tracks}

Oliver Heirich, Patrick Robertson, Thomas Strang
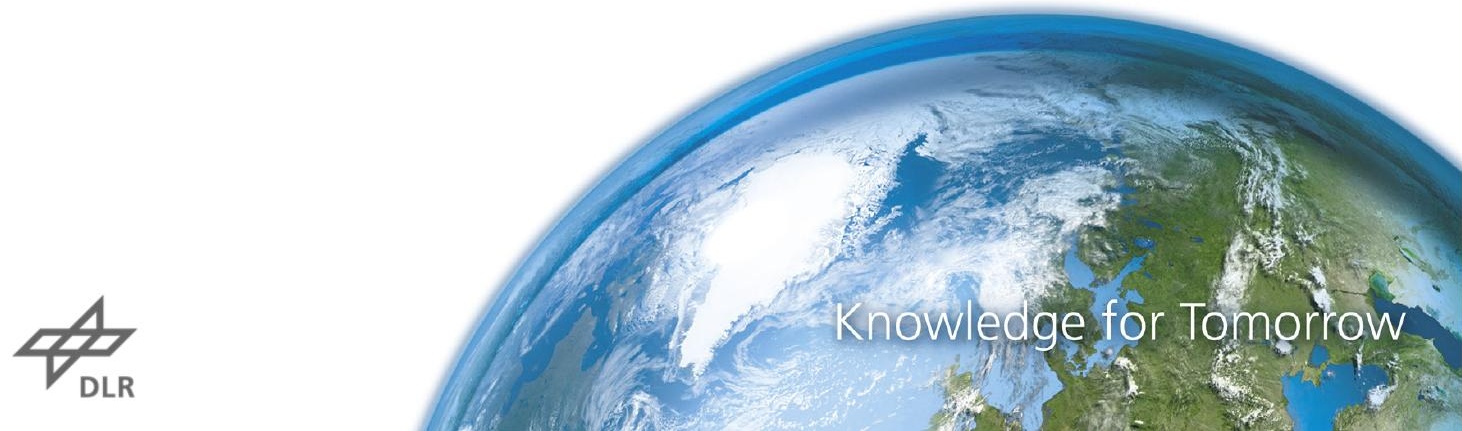


\section{Localization in Railways}

- Train localization for safety critical applications:

- General train control

- Railway collision avoidance system (RCAS)

- Semi/fully autonomous train control

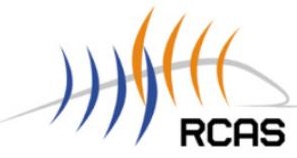

- Track selective localization

- Approach

- Train-side onboard sensors only

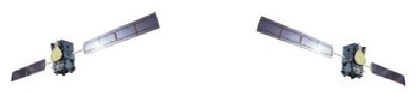

- No sensors along the tracks

- GNSS + IMU

- Map of the track network

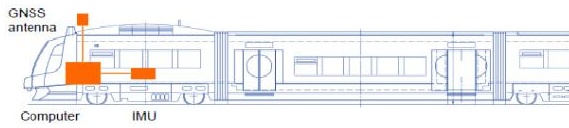




\section{Localization in Railways}

- Railway track consists of feature-rich information

- Topological connections

- Geometry
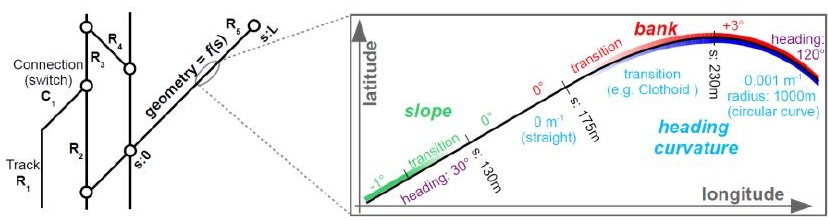

- Geometric constrains affect train kinematics, which can be measured:

$\rightarrow$ map enables topological localization with onboard sensors

How is this special map generated?

\section{$\rightarrow$ RailSLAM}

- Probabilistic, simultaneous localization and mapping algorithm

- Creation and maintenance of the probabilistic, geometric feature-rich map 


\section{How is SLAM adopted to railways?}

- SLAM phase definitions for railway scenarios:

- White space mapping phase (unknown track)

- SLAM on prior map phase (known track)

- Transitions between the phases

1. Unknown track, white space mapping

2. Reversing train, map has just been recorded

3. Train branches at a switch to unknown track

4. Train merges to a known track by switch (typical loop in railways)

5. Exceeding of known map

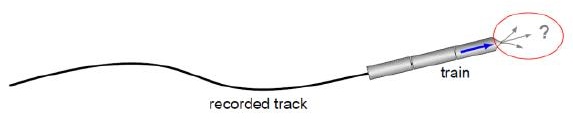

6. Circular loop closure
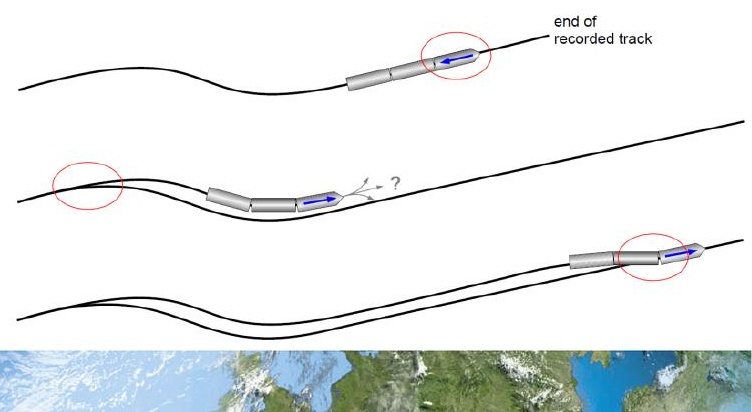


\section{SLAM scenarios - finite state machine}






\section{Probabilistic SLAM for railways}

- Dynamic Bayesian Network:

Railway specific:

Track geometry affects train kinematics which can be measured

- Joint posterior:

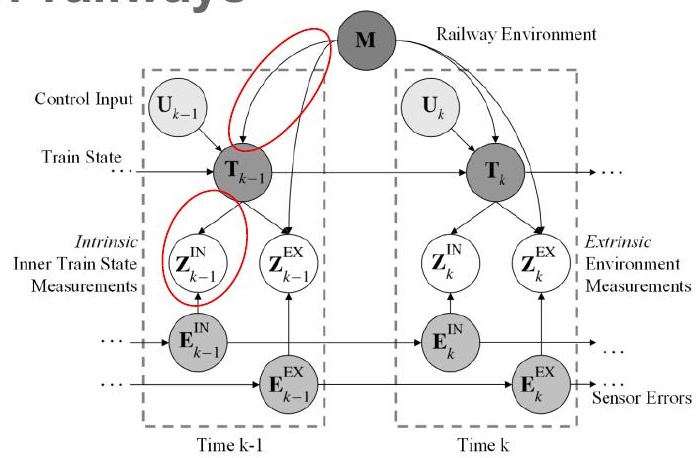

$$
p\left(\mathbf{T}_{0: k}, \mathbf{E}_{0: k}^{\mathrm{IN}}, \mathbf{E}_{0: k}^{\mathrm{EX}}, \mathbf{M} \mid \mathbf{U}_{0: k}, \mathbf{Z}_{1: k}^{\mathrm{IN}}, \mathbf{Z}_{1: k}^{\mathrm{EX}}\right)=\underbrace{p\left(\mathbf{M} \mid \mathbf{T}_{0: k}, \mathbf{Z}_{1: k}^{\mathrm{EX}}\right)}_{\text {mapping }} \cdot \underbrace{p\left(\mathbf{T}_{0: k}, \mathbf{E}_{0: k}^{\mathrm{IN}}, \mathbf{E}_{0: k}^{\mathrm{EX}} \mid \mathbf{U}_{0: k} \mathbf{Z}_{1: k}^{\mathrm{IN}}, \mathbf{Z}_{1: k}^{\mathrm{EX}}\right)}_{\text {localization }}
$$

- Factorization of the localization part $\rightarrow$ Bayesian filter in recursive formulation (particle filter):

$$
p\left(\mathbf{T}_{0: k}, \mathbf{E}_{0: k} \mid \mathbf{U}_{0: k}, \mathbf{Z}_{1: k}\right) \propto \underbrace{p\left(\mathbf{Z}_{k}^{\mathrm{IN}} \mid \mathbf{T}_{k}, \mathbf{E}_{k}^{\mathrm{IN}}\right) \cdot p\left(\mathbf{Z}_{k}^{\mathrm{EX}} \mid \mathbf{T}_{k}, \mathbf{E}_{k}^{\mathrm{EX}}\right)}_{\text {sensor measurements }} \cdot \underbrace{p\left(\mathbf{T}_{k} \mid \mathbf{T}_{k-1}, \mathbf{U}_{k}\right)}_{\text {train transition }} \cdot \underbrace{p\left(\mathbf{E}_{k} \mid \mathbf{E}_{k-1}\right)}_{\text {error transition }} \cdot \underbrace{p\left(\mathbf{T}_{0: k-1}, \mathbf{E}_{0: k-1} \mid \mathbf{U}_{0: k-1} \mathbf{Z}_{1: k-1}\right)}_{\text {previous estimate (recursion) }}
$$




\section{Part 1: Localization estimation algorithm}

- Train state $\mathrm{T}_{k}=\{\underbrace{R, s, \text { dir }}_{\text {topo position }}, \underbrace{\dot{s}, \ddot{s}}_{\text {train motion }}, \underbrace{\text { lat, lon, alt }}_{\text {geo position }}, \underbrace{\phi, \theta, \psi}_{\text {attitude }}, \underbrace{C_{\phi}, C_{\theta}, C_{\psi}}_{\text {curvatures }}\}_{k}$

- Train state estimation by EKF
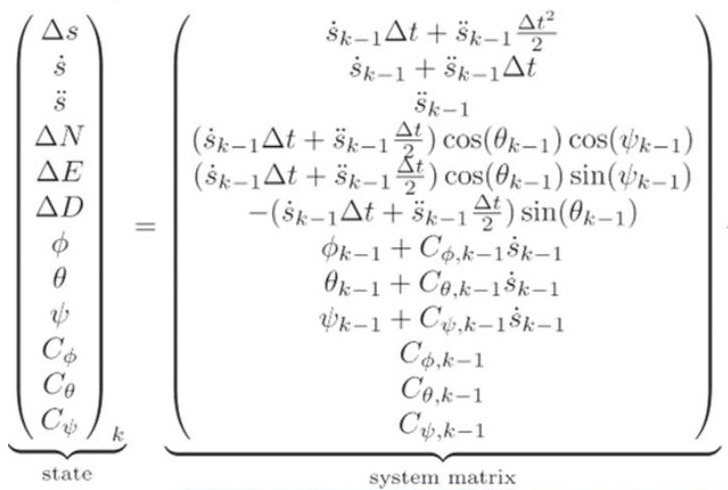

$+\underbrace{\left(\begin{array}{c}0 \\ 0 \\ \nu_{\ddot{s}} \\ 0 \\ 0 \\ 0 \\ 0 \\ 0 \\ 0 \\ \nu_{C_{\phi}} \\ \nu_{C_{\theta}} \\ \nu_{C_{\psi}}\end{array}\right)_{k-1}}_{\text {process noise }}$ 


\section{Train State EKF measurement updates}

- GNSS position and velocity updates

- Measurement of train kinematics by IMU, model contains geometry from the map (no strapdown approach)

Turn rates (gyroscopes):

$$
\begin{aligned}
& \omega^{x}=\quad C_{\phi} \dot{s} \quad-C_{\psi} \dot{s} \sin \theta \\
& \omega^{y}=\quad C_{\theta} \dot{s} \quad \cdot \cos \phi+C_{\psi} \dot{s} \sin \phi \cos \theta \\
& \omega^{z}=\underbrace{C_{\psi} \dot{s}}_{\begin{array}{c}
\text { major } \\
\text { turn rates }
\end{array}} \underbrace{\cdot \cos \theta \cos \phi-C_{\theta} \dot{s} \sin \phi}_{\begin{array}{c}
\text { attitude } \\
\text { corrections }
\end{array}} \\
& a^{x}=g \sin \theta+\overbrace{\ddot{s}}^{\text {traction }} \\
& a^{y}=-g \sin \phi \cos \theta+C_{\psi} \dot{s}^{2} \cos \phi-C_{\theta} \dot{s}^{2} \sin \phi \\
& a^{z}=\underbrace{-g \cos \phi \cos \theta}_{\text {gravity }}-\underbrace{C_{\psi} \dot{s}^{2} \sin \phi}_{\begin{array}{c}
\text { cross } \\
\text { centripetal } \\
\text { acceleration }
\end{array}}-\underbrace{C_{\theta} \dot{s}^{2} \cos \phi}_{\begin{array}{c}
\text { vertical } \\
\text { centripetal } \\
\text { acceleration }
\end{array}}
\end{aligned}
$$

Accelerations: 


\section{Part 2: Map estimation algorithm}

- Mapping:

- Track point: $\quad \operatorname{Tp}_{s}=\{\underbrace{s}_{1 \mathrm{D} \text { position }}, \underbrace{\text { lat }_{s}, l_{\text {lon }}, \text { alt }}_{\text {geo position }}, \underbrace{\phi_{s}, \theta_{s}, \psi_{s}}_{\text {attitude }}, \underbrace{C_{\phi, s}, C_{\theta, s}, C_{\psi, s}}_{\text {curvatures }}\}$

- Train state (temporal, time $k$ ) $\rightarrow$ track point (spatial, position $s$ )

- Uncertainty of map geometry (EKF covariance diagonal)

- SLAM state:

- White space mapping: creation of a new track point

- SLAM on prior map: modification of an existing track point

- Map structure:

1. Track network layer: List of tracks and connections

2. Track layer: Connections, List of track points

3. Track point layer: Geometry data and variances parameterized on the track position

- Continuous geometry data by linear interpolation between track points 


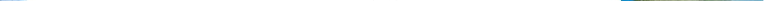




\section{Memory consumption of the map}

- Geometry: uncompressed memory estimation for the map

- Geometry: 48 byte,

- Variances: 44 byte

$$
\mathrm{Tp}_{s}=\{\underbrace{s}_{1 \mathrm{D} \text { position }}, \underbrace{\text { lat }_{s}, \operatorname{lon}_{s}, \text { alt }_{s}}_{\text {geo position }}, \underbrace{\phi_{s}, \theta_{s}, \psi_{s}}_{\text {attitude }}, \underbrace{C_{\phi, s}, C_{\theta, s}, C_{\psi, s}}_{\text {curvatures }}\}
$$

\begin{tabular}{|c|c|c|c|c|}
\hline Network & Size & Spacing: $\mathbf{1 m}$ & $\mathbf{5 m}$ & $\mathbf{1 0 m}$ \\
\hline \hline & $100 \mathrm{~km}$ & $4.6 \mathrm{MB}(8.8 \mathrm{MB})$ & $0.9 \mathrm{MB}(1.8 \mathrm{MB})$ & $0.5 \mathrm{MB}(0.9 \mathrm{MB})$ \\
\hline Germany & $63,839 \mathrm{~km} *$ & $2.9 \mathrm{~GB}(5.5 \mathrm{~GB})$ & $0.57 \mathrm{~GB}(1.1 \mathrm{~GB})$ & $0.29 \mathrm{MB}(0.57 \mathrm{~GB})$ \\
\hline EU27 & $330,892 \mathrm{~km} *$ & $15 \mathrm{~GB}(28 \mathrm{~GB})$ & $3.0 \mathrm{~GB}(5.7 \mathrm{~GB})$ & $1.5 \mathrm{~GB}(2.8 \mathrm{~GB})$ \\
\hline
\end{tabular}

track geometry (track geometry and variances)

- Topology,

- Germany 3,1 MB

- EU27 16 MB 


\section{Proof of concept (Demo with measured data)}

- Rail Vehicle Measurements: Industrial tracks of inland port

- Up to several $100 \mathrm{~m}$ recordings, $30 \mathrm{~km} / \mathrm{h}$,

- Recorded data: GNSS (GPS-Septendrio), IMU (XsensMT)

- Leica laser tachymeter reference

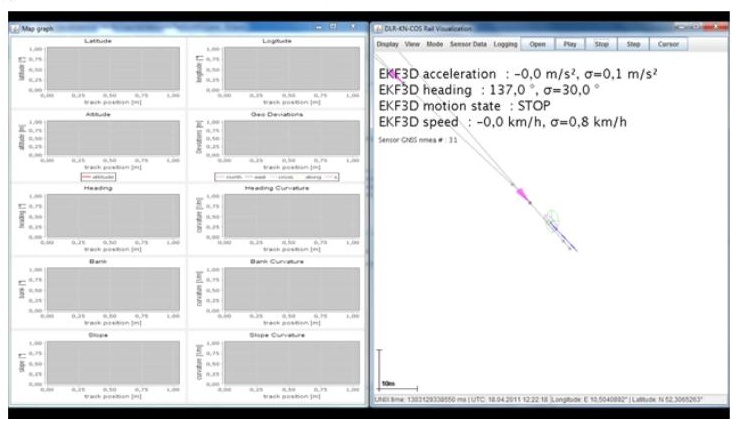




\section{Summary}

- Topological-geometric map enables train localization with onboard sensors

- We have shown a Bayesian approach of SLAM for Railways

- Mapping is enabled by: EKF Train state estimation

- Memory requirement (map): uncompressed map data of EU fits on standard storage sizes - further data compression is possible

- First results with of a feature-rich mapping with recorded data were presented

- probabilistic, geometric feature-rich map 


\section{Approach for safety critical train localization: Related, past and future work}

- Related work

- Train localization with particle filter: S. Hensel

- EKF-SLAM with spline based map for railed vehicles: C.Hasberg

- Odometry and IMU sensor fusion for trains: M.Malvezzi

- Past work

- Analysis of train/track dynamics of IMU measurements (ITSC'11)

- Bayesian multi-sensor train localization approach (IV'12)

- Train localization with particle filter on a geometric map (Fusion'12)

- Tightly coupled GNSS integration for localization

- Positioning and speed estimation by vibrations

- Ongoing and future work:

- SLAM: data association (track and position) with particle filter (FastSLAM)

- Sensor Error integration (IMU bias, GPS range bias)

- Measurement of a regional network $(>100 \mathrm{~km})$ with higher dynamics, up to $160 \mathrm{~km} / \mathrm{h}$ 


\section{RailSLAM - \\ Localization of Rail Vehicles and Mapping of Geometric Railway Tracks}

Oliver Heirich, Patrick Robertson, Thomas Strang
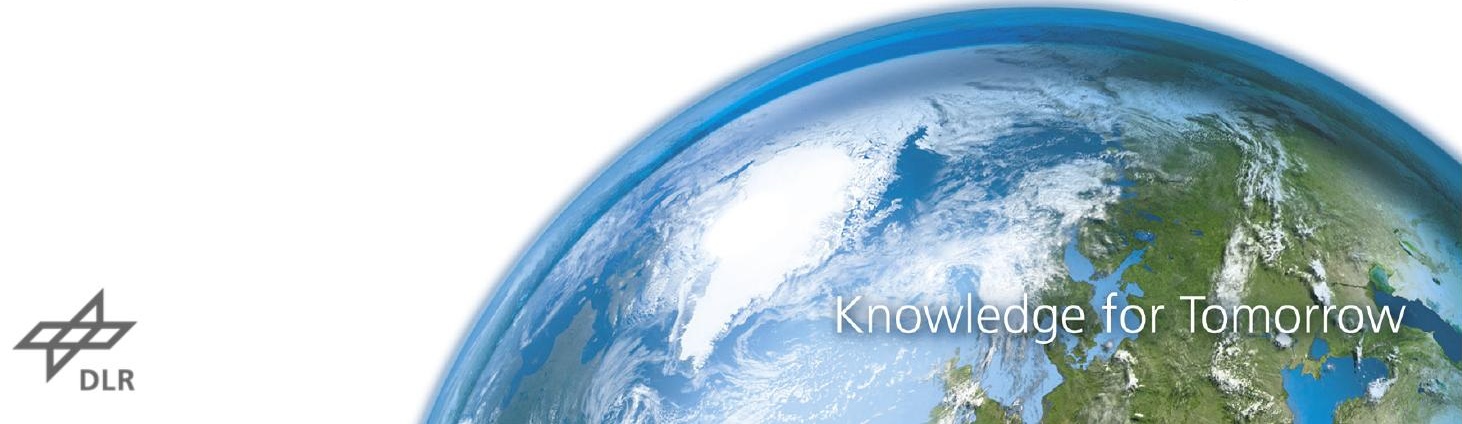